\title{
Elastic modulus and energy dissipation measurements with AFM on chalcogenide thin films
}

\author{
Attila Bonyár, Judit Kámán \\ Department of Electronics Technology \\ Budapest University of Technology and Economics \\ Budapest, Hungary \\ bonyar@ett.bme.hu
}

\author{
István Csarnovics \\ Department of Experimental Physics \\ University of Debrecen, \\ Debrecen, Hungary
}

\begin{abstract}
Contact-mode point spectroscopy and tapping-mode imaging atomic force microscopy (AFM) techniques were used to measure the Young's Modulus and tip-surface energy dissipation of amorphous chalcogenide thin films. $\mathrm{As}_{20} \mathrm{Se}_{80}$ thin film was prepared by thermal evaporation on glass substrates, and a HeNe laser $(633 \mathrm{~nm})$ was used to prepare a holographic grating on the thin film. The results from both AFM measurement techniques indicate that there are significant differences between the peaks and valleys of the grating in terms of elasticity and tipsurface energy dissipation.
\end{abstract}

Keywords - AFM, energy dissipation, chalcogenide, Young's Modulus

\section{INTRODUCTION}

It is known that illumination of the amorphous chalcogenide thin films with bandgap light can lead to significant optical and structural transformations, which can be used to create for example holographic gratings on the films. Although, these photo-induced changes are studied in great detail [1], there are little information concerning the elastic/hardness properties of the illuminated material. In this work we aim to investigate these mechanical properties of photo-induced structures in $\mathrm{As}_{20} \mathrm{Se}_{80}$ chalcogenide thin films with AFM methods.

Several techniques exist to measure the mechanical properties of surfaces with scanning probe microscopy. One possibility is to calculate the Young's Modulus of elasticity from contact-mode point spectroscopy 'force-curve' data [2], however, this only gives information regarding only one point of the sample surface. The phase images of a tapping-mode AFM, in the other hand, can give us information from a larger area of the sample. Although it is known that the contrast of a phase image during TM scan could originate from a mixture of mechanical properties - elasticity, adhesion, dissipation and deformation can also contribute to it - we tried to apply the model of Cleveland et al. to calculate the tip-surface energy dissipation based on the tapping-mode images [3]. We hope that through the application of these two methods we can obtain a concept regarding the mechanical properties of the illuminated chalcogenide material.

\section{THEORY}

\section{A. Determination of Young's modulus}

To determine Young's modulus, we applied the Hertzian model modified by Sneddon which describes the elastic indentation of a soft sample by a stiff cone [2, 4]. On an infinitely stiff sample the piezo movement equals to the cantilever deflection $d(z)$, while on a soft sample the latter decreases by the indentation depth $\delta$. Thus the indentation $\delta$ is given by the following equation:

$\delta=z-z_{0}-d$

where $z$ is the piezo position and $z_{0}$ is the $z$ position at the contact point. Since cantilever spring is linear for a small deflection, the Hook's law provides the connection between the cantilever deflection and the applied loading force of the indentation. Therefore the force equation is:

$k d=\frac{2 E \tan (\alpha)}{\pi\left(1-v^{2}\right)} \delta^{2}$

Here $k$ is the force constant of the cantilever, $E$ is the Young's modulus, $v$ is the Poisson ratio, and $\alpha$ is the half opening angle of the tip. Substituting Eq. (1) in (2) and rearranging it gives:

$z=z_{0}+d+\sqrt{\frac{k\left(1-v^{2}\right) \pi}{2 E \tan (\alpha)}} \sqrt{d}$

Based on this model two parameters can be obtained by fitting this equation on the measured contact pointspectroscopy force curves, which are $z_{0}$ and the A-factor as defined by Eq. (4).

$A=\sqrt{\frac{k\left(1-v^{2}\right) \pi}{2 E \tan (\alpha)}}$

From this Young's modulus can be expressed as Eq. (5). 
$E=\frac{k\left(1-v^{2}\right) \pi}{2 \tan (\alpha)} A^{2}$

To determine Young's modulus $(E)$ from the fitted A-factor the precise knowledge or determination of the cantilever parameters are required. For our cantilevers the nominal spring constant $(k)$ is $5 \mathrm{~N} / \mathrm{m}$, but regarding the datasheet of the supplier the variation is between $1.5 \mathrm{~N} / \mathrm{m}$ and $15 \mathrm{~N} / \mathrm{m}$. Since we do not have a method to precisely determine the force constant of the cantilevers we refrain from calculating absolute modulus values. Instead we present the measured differences as relative values compared to our selected reference, which is the surface of the $\mathrm{As}_{20} \mathrm{Se}_{80}$ chalcogenide thin film prior illumination.

\section{B. Tip-surface energy dissipation}

For the determination of tip-surface dissipation we used the model of Cleveland et al, which states that during a tappingmode scan, while the amplitude is kept constant, the phase image can strongly be related to the tip-surface energy dissipation [3]. In the most simplified case this relation can be described by Eq. (6).

$\overline{P_{\text {tlp }}}=\frac{1}{2} \frac{k A^{2} \omega_{0}}{Q_{\text {cant }}}\left[\left(\frac{A_{0}}{A}\right) \sin (\varphi)-1\right]$

Besides the measured amplitude $(A)$ and phase $(\varphi)$ of the tapping signal only the properties of the cantilever are present in the equation, such as the spring constant $(k)$, the resonance frequency $\left(\omega_{0}\right)$ and the quality factor $\left(Q_{\text {cant }}\right)$. The latter two can be obtained during the tuning of the cantilever prior the measurements.

During amplitude modulation (AM) based tapping scan the feedback loop tries to maintain a constant tapping amplitude (called setpoint), thus by presuming a well set PID controller, the variations in the amplitude signal could be considered as minimal and the $A_{0} / A$ value as constant. In this way the calculated dissipation map should reflect the variations in the phase signal only.

\section{MATERIALS AND METHODS}

\section{A. AFM measurements}

Atomic force microscope measurements were done with a Veeco (lately Bruker) diInnova type microscope in full contact and tapping mode with $512 \times 512$ sampling rate and $1 \mathrm{~Hz}$ scan rate. The PID values were optimized according to the user manual. We used Budget Sensors TAP 150Al-G probes for both contact-mode point spectroscopy and tapping-mode imaging. The nominal properties of the probes were the following: cantilever spring constant $(k) 5 \mathrm{~N} / \mathrm{m}$, resonance frequency $\left(\omega_{0}\right) 135 \mathrm{kHz}$, half cone angle $(\alpha) 20^{\circ}$, quality factor $\left(Q_{\text {cant }}\right)$ 157. (Note that the actual values were obtained before every measurement during the tuning of the cantilever). For data evaluation the freeware Gwyddion 2.27 software was used.

\section{B. Sample preparation}

$\mathrm{As}_{20} \mathrm{Se}_{80}$ chalcogenide thin films were prepared by thermal evaporation (TE) onto a glass substrate at $5 \times 10^{-6}$ mbar pressure. The composition of the samples was measured with energy dispersive X-ray spectroscopy (EDAX) using a Hitachi S-4300 system. The thickness of the samples was measured by an Ambios XP-1 profilemeter.

Holographic gratings were recorded by using two ppolarized and one additional s-polarized laser beams as this configuration has the most significant effect in chalcogenides [5]. For this purpose a He-Ne laser $(633 \mathrm{~nm})$ was used.

\section{RESULTS AND DISCUSSION}

\section{A. Determination of Young's modulus}

Contact-mode point spectroscopy measurement were done on the $\mathrm{As}_{20} \mathrm{Se}_{80}$ chalcogenide thin film to determine elastic properties of the grating. Fig. 1 presents a topography map with a corresponding cross section.
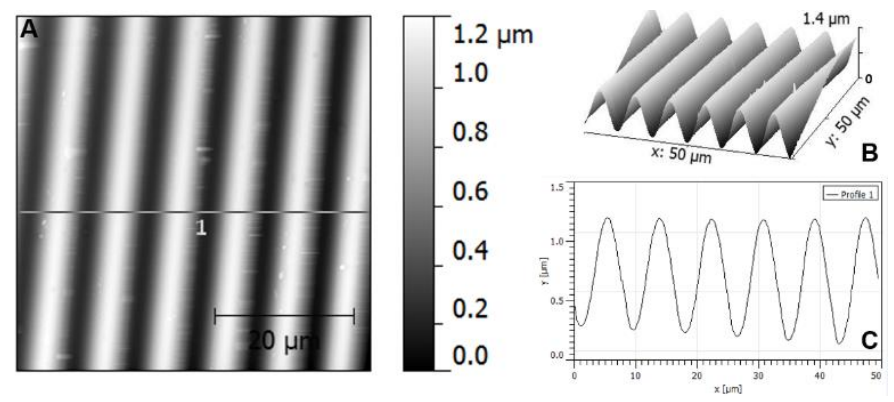

Fig. 1. Topography AFM image of the investigated optical grating (A) with 3D representation (B) and corresponding cross-section (C) at marker 1.

The force curves were obtained along one row (in the $\mathrm{x}$ lateral dimension) by using the scanners automatic position system. The precise positioning of the AFM tip is essential, since the contact between the tip and surface - the angle of incidence and the effective contact surface area - can greatly affect the force measurements. We consider the variance of contact, besides other effects, as a contributing factor to the deviation of the measurements. These other factors are the possible contaminations on the surface, and of course the "native' deviation of the elastic properties at the peaks and valleys, which can be approximated as a normal distribution.

Figures 2 and 3 present the obtained A-factors and the calculated modulus values for 20 force curves ( 10 peaks and 10 valleys, correspondingly). 


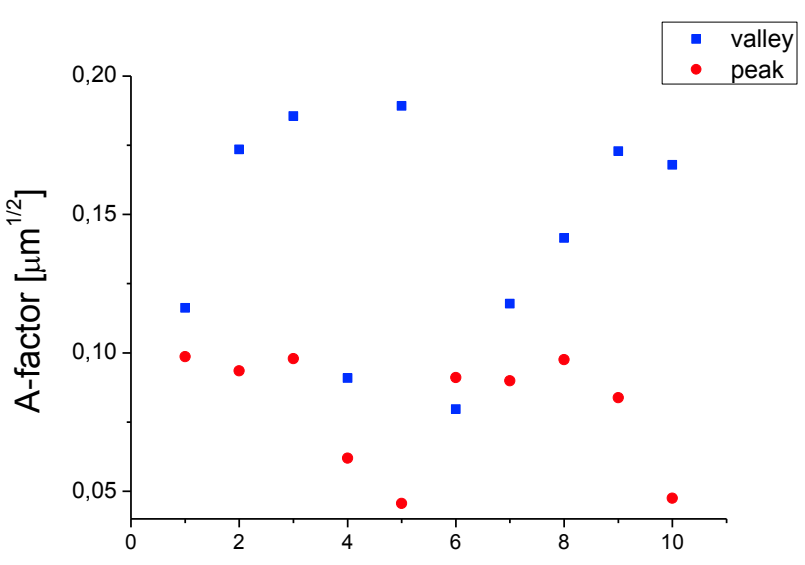

Fig. 2. A-factors obtained from contact-mode point spectroscopy measurements at the peaks and valleys of the sample.

It can be seen that, although there is deviation in the measured data, the difference in the A-factors between the peaks and valleys seem to be significant. This was confirmed by using Welch's t-test on the fitted parameters, which showed a significant bias among the A-factors of the peaks and valleys for a significance level $(p)$ of 0.01 .

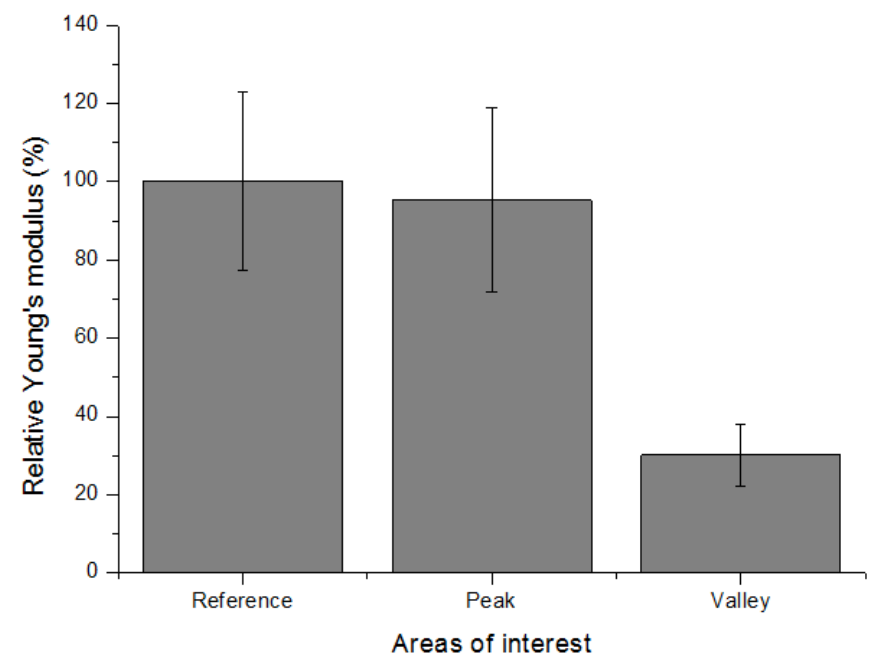

Fig. 3. Relative Young's modulus values calculated from the A-factors of Fig. 1. The relative values are compared to the mean of the reference area (which is $100 \%$ ). The number of averaged measurements are 17, 10 and 10 for the reference, peaks and valleys, respectively.

As we discussed in the theory section we refrain from the calculation of absolute modulus values due to the high variance in the cantilever parameters. Instead, in Fig 3 we present relative modulus values compared to the reference, which was measured on the chalcogenide surface, far from the illuminated area. It can be seen, that the average Young's modulus of the valleys is nearly the third of the peaks, which is close to the reference area. This means that the valleys are softer than the peaks, which can be explained by the photostructural transformations during the fabrication of the grating. Upon illumination, the structural deformation of the surface is caused by lateral mass transfer from the dark to the illuminated areas $[6,7]$. The accumulation of the material in the peaks and, in the same time, the lack of the material in the valleys may cause local density and hardness increase/decrease along the surface.

\section{B. Tip-surface energy dissipation}

To test whether Cleveland's model of tip-surface energy dissipation could be applied on our sample we made tappingmode point spectroscopy measurements at the reference area of the $\mathrm{As}_{20} \mathrm{Se}_{80}$ chalcogenide thin film. Fig. 4 presents the resulting amplitude and phase signals and also the tip-surface dissipation, which was calculated by Eq. 5. The red bars indicate the range of the amplitude signal during a closed loop AM mode scan, while the feedback tries to maintain a constant amplitude and thus only minimal variation may occur. During our subsequent measurements we used an amplitude setpoint $\left(A_{s} \sim A\right)$ that our approximately constant $A / A_{0}$ value was 0.8 . It can be seen that in this region (highlighted by the red bars) the calculated dissipation has small sensitivity to the variations in the amplitude, and thus we can presume that it will be primarily depending on the material properties of the surface.

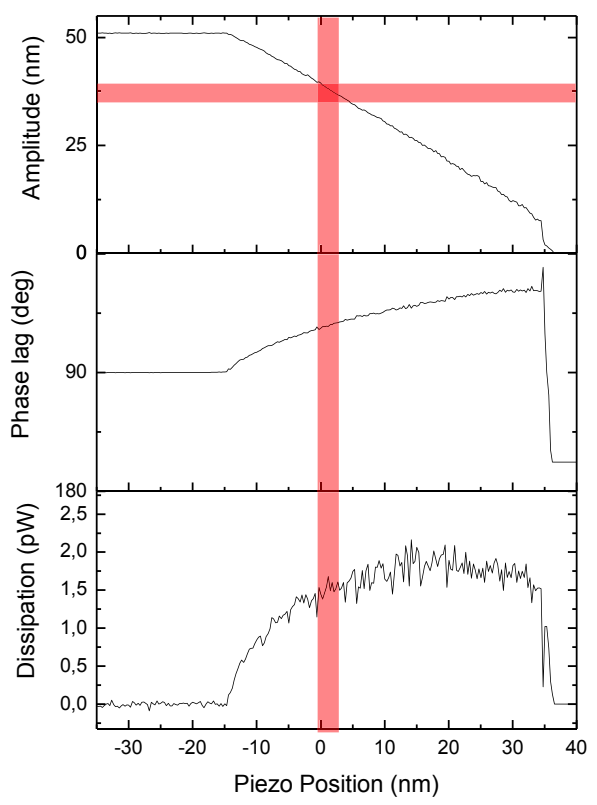

Fig. 4. A typical tapping-mode point spectroscopy with the calculated tipsurface energy dissipation measured on the reference area of the sample. Red bars indicate the amplitude range during a closed loop AM scan.

Fig. 5 shows corresponding TM topography, amplitude, phase and calculated energy dissipation maps for the investigated area. 
2014 IEEE 20th International Symposium for Design and Technology in Electronic Packaging (SIITME)
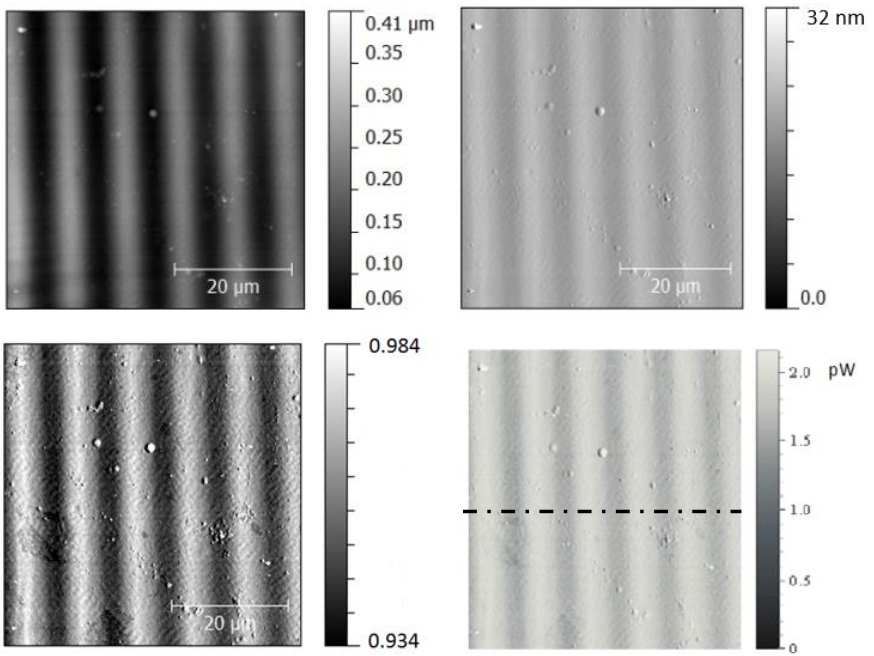

Fig. 5. Tapping-mode AFM topography (top left), amplitude (top right), sinus phase (bottom left) and calculated tip-surface dissipation (bottom right) maps.

The phase map (bottom left image) clearly shows sharp contrast between the peaks and valleys of the grating. We are aware that several factors can contribute to the contrast in the phase signal, and thus it cannot be stated that it reflects only the variation in the elastic properties of the surface. However, the calculated dissipation map correlates with the previously obtained Young's modulus, namely, the valleys have lower dissipation compared to the peaks, which are closer to the dissipation measured on the reference area (around $1.9 \mathrm{pW}$ ).

A cross-section of the dissipation map is presented in Fig 6. The differences between the tip-surface energy dissipation at the peaks and valley can clearly be seen.

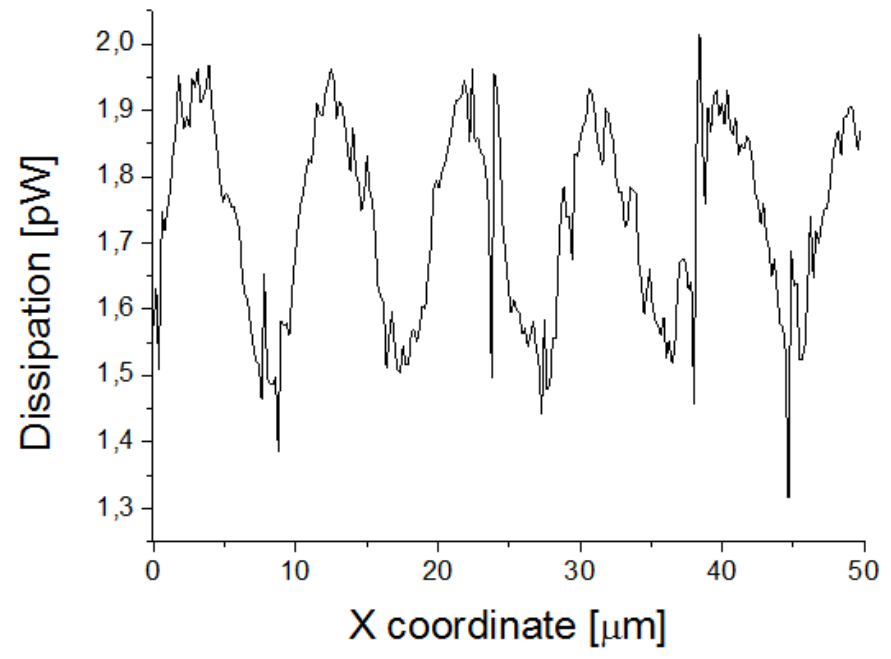

Fig. 6. Cross-section of the calculated dissipation map at the indicated marker in Fig 5.

\section{CONCLUSIONS}

Contact-mode point spectroscopy and tapping mode AFM images were made on $\mathrm{As}_{20} \mathrm{Se}_{80}$ chalcogenide thin films in order to measure the mechanical properties of a holographic grating. We found that there is a significant difference in the Young's modulus and tip-surface energy dissipation between the peaks and valleys of the grating - namely we found the valleys to be softer than the peaks and reference area -, which can be a possible consequence of the lateral mass transfer occurring during illumination. For the best of our knowledge, this is the first time that such mechanical differences were observed on a chalcogenide thin film grating, which was fabricated by optical illumination.

\section{ACKNOWLEDGMENT}

This research was supported by the European Union and the State of Hungary, co-financed by the European Social Fund in the framework of TÁMOP 4.2.4. A/2-11-1-2012-0001 'National Excellence Program'. A. Bonyár is grateful for the support of Bolyai János Scholarship and the Robert Bosch Scholarship.

\section{REFERENCES}

[1] A. Kikineshi, V. Palyok, M. Shiplyak, I. A. Szabó, D. L. Beke, "Photo-induced surface deformation during hologram recording in a-Se films", Journal of Optoelectronics and Advanced Materials, Vol. 2, No. 1, pp. 95-98, March 2000;

[2] J. Domke, and M. Radmacher. "Measuring the elastic properties of thin polymer films with the AFM",.Langmuir, Vol. 14, pp. 3320-3325, 1998;

[3] J. P. Cleveland, B. Anczykowski, A. E. Schmid, and V. B. Elings, „Energy dissipation in tapping-mode atomic force microscopy", Applied Physics Letters, Vol. 72. No. 20, pp. 2613-2615, May 1998;

[4] H. J. Butt, B. Cappella, M. Kappl ,"Force measurements with the atomic force microscope: Technique, interpretation and applications", Surface Science Report, Vol. 59 (1-6), 1-152, November 2005;

[5] U. Gertners, J. Teteris, "Surface relief formation in amorphous chalcogenide thin films during holographic recording", Optical Mat. Vol. 32 807-810, 2010;

[6] Yu. Kaganovskii, D. L. Beke, Charnovych, Kökényesi, and M. Trunov, "Inversion of the direction of photo-induced mass transport in As20Se80 films: Experiment and theory" Journal of Applied Physics, Vol. 110 063502, 2011;

[7] M. Trunov, P. Lytvyna V. Takats I. Charnovych, S. Kökenyesi, "Direct surface relief formation in As0.2Se0.8 layers", Journal of Optoelectronics and Advanced Materials, Vol. 11, No. 12, pp. 1959 - 1962, December 2009. 